\title{
Atomic Motion in Amorphous Solids
}

\author{
H.R. SCHOBER \\ Institut für Festkörperforschung, Forschungszentrum Jülich \\ 52425 Jülich, Germany
}

\begin{abstract}
Glasses and amorphous materials show, coexisting with the sound waves, a variety of low energy excitations: tunneling, quasi-localized vibrations and relaxations. The latter two are observed well into the liquid state. Using molecular dynamics both were shown to be centred on more than ten atoms or molecular units, which form chain-like structures. With increasing frequency the interaction of the quasi-localized modes with the sound waves and with each other increases, they delocalize. However, even at the so-called boson peak frequency, where the sound waves become overdamped due to the interaction, the vibrations can be decomposed into local and extended modes. Closely correlated with the local vibrations are the local relaxations, which can be envisaged as collective jumps of groups of atoms. With rising temperature both the total jump length and the number of atoms participating increases. In the melt when single jumps are no longer resolved one still observes a collective motion of chains of atoms.
\end{abstract}

PACS numbers: 61.43.Fs, 61.20.Lc, 81.05.Kf

\section{Introduction}

Glasses and amorphous solids are materials without long range order of their constituents. They have, however, a considerable short range order which decays on the scale of a few nearest neighbour distances [1]. There is no universally agreed distinction between glasses and other amorphous substances. Some workers speak of glasses only when the liquid is quenched beyond its melting point into the undercooled region, until finally the transition from liquid to glass is seen as change of the volume expansion or as step in the specific heat. The corresponding glass transition temperature, $T_{\mathrm{g}}$, is not a well defined quantity, it depends on the history of the glass, especially the quench rate. Sometimes the glass transition is defined as the temperature where the viscosity grows to $\eta\left(T_{\mathrm{g}}\right)=10^{13}$ Poise. The values of $T_{\mathrm{g}}$ obtained by different definitions vary by a few percent. Apart from this quench procedure amorphous materials can be produced by various other techniques such 
as irradiation. One can expect many similarities between defect crystals and amorphous materials. In the following we will not distinguish between glasses and other amorphous materials.

At a first view glasses behave like crystalline solids. They can carry sound waves, their elastic constants are similar to the ones of their crystalline counterparts (albeit isotropic) and the vibrational spectra are similar. At low temperatures, however, qualitative differences are observed.

At the lowest temperatures, below $\approx 1 \mathrm{~K}$, the dynamics in glasses is dominated by two-level systems [2] which can be described by the tunneling model which was formulated nearly thirty years ago $[3,4]$. In addition to these tunneling states, one observes in glasses local relaxations and at frequencies near $1 \mathrm{THz}$ an excess of vibrations over the Debye value (given by the sound waves), the so-called boson peak. To describe these excess of low energy excitations, the tunneling model was extended to the soft potential model $[5,6]$. From this model "universal" temperature dependencies can be derived for temperatures of some $10 \mathrm{~K}$. Comparing the model to experiment one finds that the excess low energy excitations are collective motions of 10 to 100 atoms $[7,8]$.

The most popular model to describe the undercooled liquid is the mode coupling theory [9]. In this statistical model a nonlinear memory kernel leads to a blocking of modes at $T_{\mathrm{c}}$. It predicts scaling relations and dependencies of the form $\left(T-T_{\mathrm{c}}\right)^{\gamma}$ for quantities such as the diffusion constant. The temperature $T_{\mathrm{c}}$ is normally well above $T_{\mathrm{g}}$. In the so-called fragile glasses (metallic glasses, polymers) it is seen in a sharp upturn of the viscosity in the undercooled liquid.

At present there is not yet a common theory for both the glassy and the supercooled state. The transition from one regime into the other is probed by experiment and increasingly by computer simulations. The accumulated data should help to probe the limits of the existing theories and guide towards a theory bridging the present gap between glass and liquid theories.

In this contribution we will review computer simulations mainly in the glass. The emphasis will be on collective effects.

\section{Simulation models}

Our molecular dynamics simulations are done for systems of 500 to 10000 atoms with periodic boundary conditions. The equations of motion are integrated by the velocity Verlet algorithm with a time step of order fs. We use cooling rates of $\geq 10^{11} \mathrm{~K} / \mathrm{s}$ and aging times of order ns. For the model systems of soft spheres and Lennard-Jones atoms these values correspond to a conversion to Ar. Zero external pressure was exerted on all but the soft sphere systems where the volume was kept constant. Details of the simulations can be found in the references given further down.

To check the material dependence of the results it is necessary to simulate a variety of different materials. In our group we use model systems (soft spheres and 
Lennard-Jones, both monoatomic and binary) and models of the binary metallic glass CuZr and of Se.

The soft spheres (SSG) are described by a purely repulsive potential

$V(R)=\epsilon(\sigma / R)^{n}+V_{\text {cut }}$,

where $n=6$ or $n=12$ and $V_{\text {cut }}$ is a small correction to give a smooth cutoff.

As a simple model potential which allows for zero external pressure we use a Lennard-Jones potential

$$
V(R)=\epsilon\left[(\sigma / R)^{12}-(\sigma / R)^{6}\right]+V_{\text {cut }} .
$$

We use this potential both for monoatomic and binary systems. In the latter the parameters are taken from Kob and Andersen [10].

As a typical example of a binary metallic glass we chose $\mathrm{Cu}_{33} \mathrm{Zr}_{67}$. The interaction is described by a modified embedded atom potential, for details see [11]. The results do not strongly depend on the choice of interaction. As far as data are available there is a good agreement with the similar NiZr system modelled with a totally different interaction [12].

As an example of a good monoatomic glass former we simulate selenium. Se has a coordination number near 2. The amorphous structure consists of internetted rings and chains. We describe it by a potential of the Stillinger-Weber type. In this potential the covalent bonds are described by an additional three-body term $V_{3}\left(\left|\boldsymbol{R}^{1}-\boldsymbol{R}^{2}\right|,\left|\boldsymbol{R}^{1}-\boldsymbol{R}^{3}\right|, \cos \Theta_{213}\right)$, where $\Theta_{213}$ is the apex angle [13].

The examples presented in the following will be taken from simulations of these different materials. We will emphasize the qualitative aspects which are equal for all these systems.

\section{Vibrations in the glass}

The vibrations in a glass at low temperatures can be most easily studied in the harmonic approximation. As example we quench samples of up to 5488 atoms of a SSG with $n=6$ (Eq. (1)) to $T=0 \mathrm{~K}$. The atomic configuration will then correspond to a minimum of the potential energy and we can expand the energy in terms of the displacements from this minimum energy. The quadratic term defines a dynamical matrix

$$
D_{\alpha \beta}^{i j}=\frac{1}{\sqrt{m_{i} m_{j}}} \frac{\partial^{2} E_{\mathrm{pot}}(\{\boldsymbol{R}\})}{\partial R_{i, \alpha} \partial R_{j, \beta}} .
$$

Here we denote by $i, j$ the atoms and by $\alpha, \beta$ the Cartesian coordinates. Diagonalization gives, in harmonic approximation, the frequencies of the eigenmodes of vibration and their eigenvectors, i.e. their spatial structure. Figure 1 shows for the SSG the spectrum of all modes as well as the Debye spectrum, calculated from the elastic constants. The area between the two curves corresponds to the excess in low frequency modes, typical of the glassy structure. In a plot of $Z(\nu) / \nu^{2}$ one finds a maximum around $\nu=0.1$, the boson peak. 


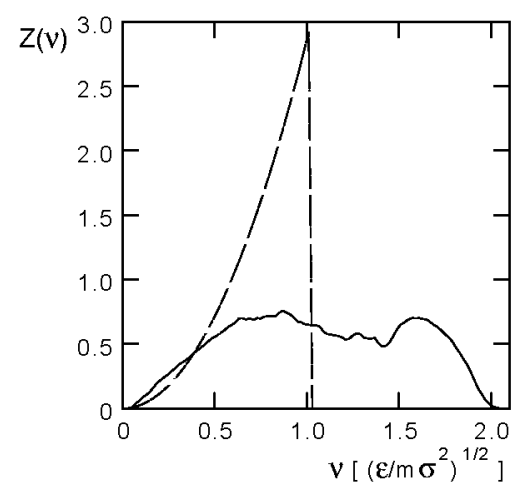

Fig. 1. Configurationally averaged vibrational density of states of the soft sphere glass (solid line) with $n=6$ and Debye spectrum (dashed line) [14].

The eigenmodes at the boson peak frequency have a complicated structure. This has to be expected, since low frequency vibrations are resonant modes and not proper localized modes. Localized modes are observed at the high frequency end of the spectrum. The low frequency modes can, however, be decomposed into sound waves and local vibrations [14]. The latter are in full accord with the predictions of the soft potential model [7]. The interaction with the local vibrations causes an attenuation of the sound waves. With increasing frequency the Ioffe-Regel limit is reached, the phonon mean free path drops to the wavelength. The increasing interaction between the modes leads to a level repulsion and $Z \nu \propto \nu$. In the $Z(\nu) / \nu^{2}$ representation the intensity drops causing the boson peak maximum.

The cores of the deconvoluted soft localized modes form chain-like structures with side branching and have a dimension $\approx 1.5$. The modes are centred at irregularities of the glass structure. The first neighbour shell is reduced in number but compressed, i.e. the modes are not connected with holes in the glass.

The soft quasi-local vibrations originate in the frustration inherent to the disorder. For a simple one-atomic densely packed structure one can envisage the frustration stemming from the difference of the globally most dense structure (fcc or hcp) and the locally most dense icosahedral structure. The latter structure is not space filling. The resulting structural compromise will lead to some atoms or groups of atoms being squeezed into positions belonging to neither structure.

We illustrate this for an example of a quasi-localized soft mode. The atoms are shifted along the direction of the $3 N$-dimensional, normalized eigenvector, $\boldsymbol{e}$, according to

$$
\boldsymbol{R}^{n}(x)=\boldsymbol{R}_{0}^{n}+x \boldsymbol{e}^{n},
$$

where $\boldsymbol{R}_{0}^{n}$ is the equilibrium position of atom $n$ [15]. For simplicity we have not normalized the amplitude $x$ to an effective atomic amplitude as is usually done in the soft potential model. In Fig. 2 the average potential energy per atom, $\langle U(x)\rangle_{\text {tot }}$ (solid line), is shown as a function of the displacement along a single 


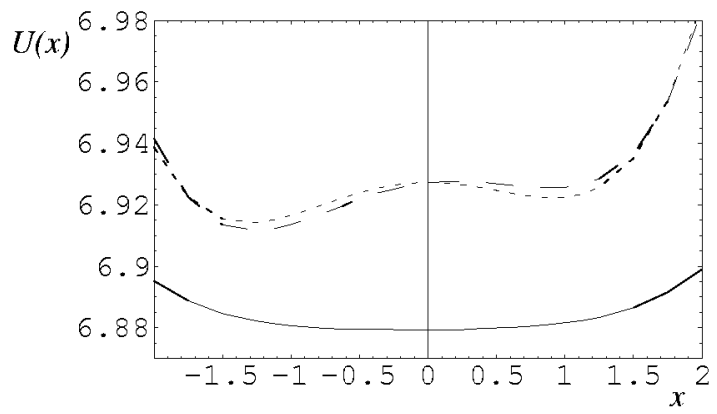

Fig. 2. Average potential energy of atoms in a single soft mode with frequency $\nu=0.0985$, participation ratio $p=0.23$. Solid line: $\langle U(x)\rangle_{\text {tot }}$, potential energy averaged over all atoms in the system. Dashed line: $\langle U(x)\rangle_{\text {core }}$, the partial potential energy, averaged over 61 atoms of the core of the mode. Dotted line: least squares fit of the partial potential energy by a soft potential polynomial $U^{*}(x)=A x^{2}+B x^{3}+C x^{4}+\langle U(0)\rangle_{\text {core }}$ [15].

soft eigenmode. This is one of the soft potentials which are described by the soft potential model $[16,17]$. By the dashed line the average potential energy of the 61 atoms which are most active in the given mode, $\langle U(x)\rangle_{\text {core }}$ are shown. Atoms are considered as active in a given mode if their amplitude, $\left|e^{n}\right|$, is at least $30 \%$ of the maximal atomic amplitude in the mode. The partial potential energy of the core atoms is indeed double-well shaped with minima at $x_{\mathrm{m}} \approx \pm 1.3$, which corresponds to maximal displacements of individual atoms by $\left|\boldsymbol{R}^{n}-\boldsymbol{R}_{0}^{n}\right| \approx(0.2-0.3) \sigma$ from the equilibrium configuration. This maximal atomic displacement is of the order of the one observed in local low temperature relaxations [18]. The atoms are stabilized in their minimum position by the embedding into the glassy matrix. This situation is very similar to what one observes for self-interstitial atoms in fcc metals [19].

Similar low frequency modes have been observed in simulations of $\mathrm{SiO}_{2}[20]$, $\mathrm{Se}$ [21], $\mathrm{B}_{2} \mathrm{O}_{3}$ [22], in $\mathrm{Ni}-\mathrm{Zr}$ [23] and $\mathrm{Pd}-\mathrm{Si}$ [24], in amorphous ice [25] and in amorphous and quasi-crystalline $\mathrm{Al}-\mathrm{Zn}-\mathrm{Mg}[26]$.

\section{Relaxations in glasses}

In addition to these periodic excitations, aperiodic thermally activated relaxations of the structure occur. In the soft potential model the local relaxations (at low temperatures) are strongly correlated with the local soft vibrations. Experimentally this is supported by the similarity of the structure factors of both types of excitations [27]. At low temperatures these local relaxations can be envisaged as a change of configuration by thermally activated hopping of a group of atoms. These configurations are confined to some area of configuration space separated from the rest by higher barriers. With rising temperature higher barriers can be overcome until finally diffusion becomes possible. From diffusion measurements in 
metallic glasses effective masses of more than ten have been deduced for the group of atoms participating in the jump processes [28].

To study relaxations we heat the glasses from $T=0 \mathrm{~K}$ to the desired temperature where they are kept approximately for $0.5 \mathrm{~ns}$. After an initial equilibration time of $\approx 50 \mathrm{ps}$ at each temperature we monitor the total displacement $\Delta R$ from the starting configuration [29]

$$
\Delta R(t)=\sqrt{\sum_{n}\left[\boldsymbol{R}^{n}(t)-\boldsymbol{R}^{n}(0)\right]^{2}},
$$

where $\boldsymbol{R}^{n}(t)$ is the position vector of particle $n$ at time $t$ and $\boldsymbol{R}^{n}(0)$ is the one at the starting or reference configuration. $\Delta R(t)$ oscillates due to the vibrations and changes due to relaxations, i.e. due to the transitions from one local energy minimum to another. An example is shown in Fig. 3 for the SSG at two temperatures. At $T=0.02 T_{\mathrm{g}}$ the glass clearly jumps between three configurations. Let us denote the configurations $A, B, C$. The jump sequence is $B \rightarrow C \rightarrow B \rightarrow C \rightarrow A \rightarrow C \rightarrow A$. By quenching to $T=0 \mathrm{~K}$ we find the potential energy differences $\Delta E^{B}-\Delta E^{A}=1.74 \times 10^{-3} \epsilon$ and $\Delta E^{C}-\Delta E^{A}=1.81 \times 10^{-3} \epsilon$. These energy differences are of the order of the temperature. The corresponding spatial distances between the configurations, Eq. (5), are $\Delta R^{A-B}=1.63 \sigma$, $\Delta R^{A-C}=0.96 \sigma$, and $\Delta R^{B-C}=1.0 \sigma$, i.e. they are of the order of the nearest neighbour distance $R_{\mathrm{NN}}=1.1 \sigma$. The maximal distance an individual atom travels in these jumps is only $0.3 \sigma$, about a quarter of $R_{\mathrm{NN}}$. Such relaxations can be observed experimentally e.g. as telegraph noise in the electric resistivity of point contacts [30]. Increasing the temperature by a factor of four the average displacement in the initial configuration doubles as expected for a vibrational mean square displacement. The jumps seen at the lower temperature can no longer be resolved and new jumps over larger distances are observed.

All observed relaxations are collective jumps localized to 10 or more atoms forming twisted chain-like structures with some side branching [18]. An example of such a structure is shown in Fig. 4 [31]. The chain structure is a consequence of the dominance of the nearest neighbour bond in close packed structures. A low temperature relaxation is only possible if these bonds are not strongly compressed. We observe similar structures in amorphous Se [29, 30] and CuZr [11, 32].

In materials with a different inherent structure these relaxations will be different. In $\mathrm{SiO}_{2}$ we would expect them to be collective twists of tetrahedra as has been postulated for the soft vibrations [33]. The origin will be, however, the same as for the soft vibrations, namely local stresses leading to a softness in one direction of the multidimensional configuration space.

When the temperature is increased, the jump width increases. This is plausible considering that the relevant barriers will become higher. At the same time also the number of atoms participating in a single jump increases, the jumps become more collective [34]. 


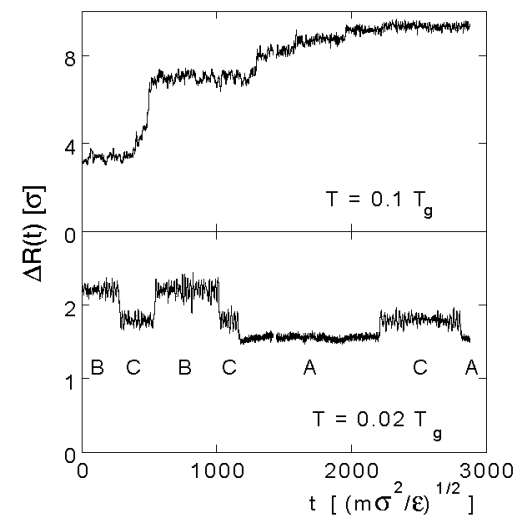

Fig. 3. Total displacement relative to a local minimum configuration as function of time for one sample of the glass of Fig. 1 with $N=5488$ at two temperatures. Please note the different scales for $\Delta R[18]$.

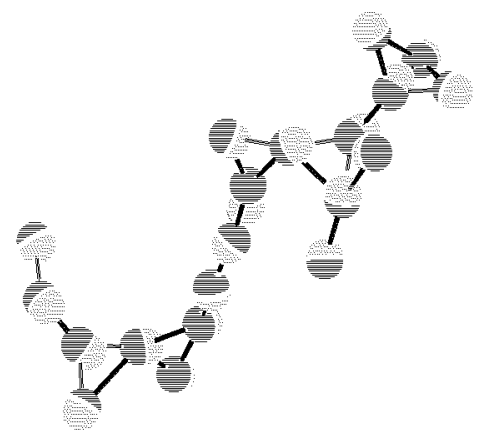

Fig. 4. Collective jump in the glass of Fig. 1 at $T=0.15 T_{\mathrm{g}}$. The initial positions of the atoms are shown by the dark spheres and the final ones by the shaded spheres. The bonds connect nearest neighbours. Shown are all atoms with more than $40 \%$ of the maximal atomic displacement [31].

In accord with the soft potential model, the local relaxations are strongly correlated with the soft vibrations $[29,18]$. There is also a strong correlation between subsequent jumps. If one marks the atoms which have jumped during a time interval this latter correlation leads to an increase in time in the number of "movable atoms". This effect is known as dynamic heterogeneity. We illustrate it in Fig. 5 where we show the mobile atoms at $T \approx 0.157 T_{\mathrm{g}}$ after some ns (in $\mathrm{Ar}$ units). The effect is general, the size of the region at a given time and temperature will depend on the particulars of the interatomic interaction. The dynamic heterogeneity is largest at low temperatures and rapidly decreases in the undercooled liquid. In a glass at low enough temperatures it persists "for ever". At higher temperatures all atoms will move eventually, and homogeneity is restored for long 


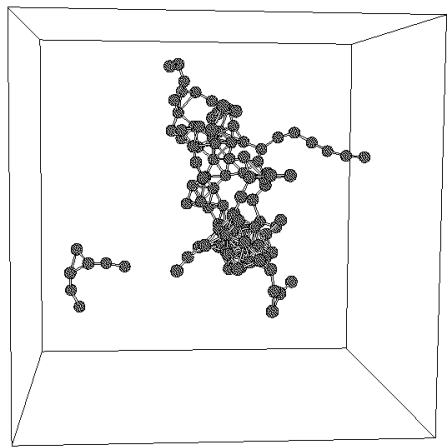

Fig. 5. Initial positions in a sample of the glasses of Fig. 1 of atoms displaced by more than 0.3 of the average nearest neighbour distance during a time interval $\Delta t=3600\left(m \sigma^{2} / \epsilon\right)^{1 / 2}$ at $T \approx 0.15 T_{\mathrm{g}}$. The total displacement is approximately 8 nearest neighbour distances [18].

times.

The correlation between subsequent jumps is seen also in burst of activity. A spatial region of the glass can be inactive for a long time. But one jump can trigger a number of correlated jumps before the glass settles again into a more inactive period [35].

\section{Relaxations in the melt}

Computer simulations in the liquid state, especially with respect to MCT, have been discussed in numerous papers and reviews, see e.g. [10, 12, 36]. For simple systems the predictions of MCT are at least semiquantitatively reproduced in these calculations.

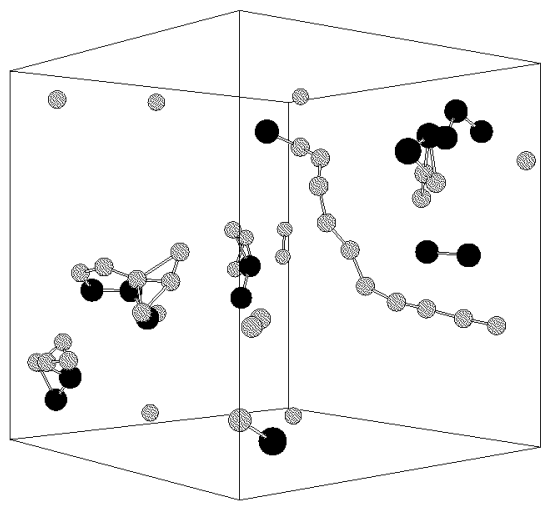

Fig. 6. Atoms in a melt of $\mathrm{Cu}_{33} \mathrm{Zr}_{67}$ at $T=1200 \mathrm{~K}$ with the largest displacements between time averaged configurations separated by $6.5 \mathrm{ps}$. Shown are Cu (light spheres) and Zr (dark spheres) atoms displaced by more than $1.6 \AA$ and $1.45 \AA$, respectively [34]. 
In the liquid state it is no longer possible to separate single jumps. To obtain information on the atomic structure of the motion one can study the difference between structures some ps apart. To remove the effects of vibrations these configurations have to be averaged over a typical vibrational period. Figure 6 shows an example for undercooled liquid $\mathrm{Cu}_{33} \mathrm{Zr}_{67}$. There are two striking effects. First, clearly the smaller $\mathrm{Cu}$ atoms are much more mobile. Second, we observe string-like structures similar to the ones shown for the amorphous materials, Figs. 3 and 4 . These structures indicate a high degree of collectivity in the motion also in the undercooled liquid. They have been studied extensively for a binary Lennard-Jones system by Donati et al. [37]. These authors report a marked increase in collectivity when the liquid is quenched to $T_{\mathrm{c}}$. These mobile strings again will lead to a dynamic heterogeneity. Experimentally collective motion has also been deduced from the vanishing isotope effect in diffusion [38]. In the simulation one observes a decreasing isotope effect when one cools towards $T_{\mathrm{g}}[39]$.

\section{Summary}

In summary, the simulations have shown that the additional vibrational modes observed in glasses at low frequencies are due to quasi-localized vibrations. These interact with the sound waves and with each other. This leads to a strong mixing of the modes, a delocalization. The computer simulation supports the description in terms of the soft potential model. The modes are typically centred on around 20 atoms. The localized vibrations are related to irregularities of the glass structure, causing some frustration. The modes stripped of their interaction tend to low dimensional, chain-like structures. Closely related to the soft localized vibrations are local relaxations which can be understood as a collective hopping of atoms. In the investigated systems these groups of atoms form again low dimensional structures, typically chains with some side branches.

With increasing frequency the interaction between the localized low frequency vibrations increases leading to a increased delocalization of the modes. Similarly the number of atoms participating in the hopping motion increases with temperature. There is a strong correlation between hopping and local vibrations and a long-lived correlation between subsequent hops.

\section{Acknowledgments}

Part of this work was financially supported by Deutsche Forschungsgemeinschaft, Schwerpunkt "Unterkühlte Metallschmelzen: Phasenselektion und Glasbildung". 


\section{References}

[1] Glasses and Amorphous Materials, Vol. 9 of Materials Science and Technology, Ed. J. Zarzycki, VCH, Weinheim 1991.

[2] Amorphous Solids: Low Temperature Properties, Ed. W.A. Phillips, Springer-Verlag, Berlin 1981.

[3] W. Phillips, J. Low Temp. Phys. 7, 351 (1972).

[4] P.W. Anderson, B.I. Halperin, C.M. Varma, Philos. Mag. 25, 1 (1972).

[5] V.G. Karpov, M.I. Klinger, F.N. Ignatiev, Sov. Phys. JETP 57, 439 (1983).

[6] M.A. Il'in, V.G. Karpov, D.A. Parshin, Sov. Phys. JETP 65, 165 (1983).

[7] U. Buchenau, Y.M. Galperin, V.L. Gurevich, H.R. Schober, Phys. Rev. B 43, 5039 (1991).

[8] U. Buchenau, Y.M. Galperin, V.L. Gurevich, D.A. Parshin, M.A. Ramos, H.R. Schober, Phys. Rev. B 46, 2798 (1992).

[9] W. Götze, A. Sjölander, Rep. Prog. Phys. 55, 241 (1992).

[10] W. Kob, H. Andersen, Phys. Rev. E 51, 4626 (1995).

[11] C. Gaukel, Berichte des Forschungszentrums Jülich, Vol. 3556, Forschungszentrum Jülich, Jülich 1998.

[12] H. Teichler, Phys. Rev. Lett. 76, 62 (1996).

[13] C. Oligschleger, R.O. Jones, S.M. Reimann, H.R. Schober, Phys. Rev. B 53, 6165 (1996).

[14] H.R. Schober, C. Oligschleger, Phys. Rev. B 53, 11469 (1996).

[15] V.A. Luchnikov, N.N. Medvedev, Y.I. Naberukhin, H.R. Schober, Phys. Rev. B 62, $3181(2000)$.

[16] V.G. Karpov, M.I. Klinger, F.N. Ignatiev, Sov. Phys. JETP 57, 439 (1983).

[17] M.A. Il'in, V.G. Karpov, D.A. Parshin, Sov. Phys. JETP 65, 165 (1983).

[18] C. Oligschleger, H.R. Schober, Phys. Rev. B 59, 811 (1999).

[19] P. Ehrhart, K.H. Robrock, H.R. Schober, in: Physics of Radiation Defects in Crystals, Eds. R.A. Johnson, A.N. Orlov, North-Holland, Amsterdam 1986, p. 3.

[20] W. Jin, P. Vashishta, R.K. Kalia, J.P. Rino, Phys. Rev. B 48, 9359 (1993).

[21] C. Oligschleger, H.R. Schober, Physica A 201, 391 (1993).

[22] R. Fernández-Perea, F.J. Bermejo, E. Enciso, Phys. Rev. B 53, 6215 (1996).

[23] J. Hafner, M. Krajci, J. Phys., Condens. Matter 6, 4631 (1994).

[24] P. Ballone, S. Rubini, Phys. Rev. B 51, 14962 (1995).

[25] M. Cho, G.R. Fleming, S. Saito, I. Ohmine, R.M. Stratt, J. Chem. Phys. 100, 6672 (1994).

[26] J. Hafner, M. Krajci, J. Phys., Condens. Matter 5, 2489 (1993).

[27] U. Buchenau, H.M. Zho, N. Nücker, K.S. Gilroy, W.A. Phillips, Phys. Rev. Lett. 60, 1318 (1988).

[28] F. Faupel, P.W. Hüppe, K. Rätzke, Phys. Rev. Lett. 65, 1219 (1990).

[29] C. Oligschleger, H.R. Schober, Solid State Commun. 93, 1031 (1995). 
[30] V.I. Kozub, C. Oligschleger, J. Phys., Condens. Matter 10, 8033 (1998).

[31] H.R. Schober, C. Oligschleger, B.B. Laird, J. Non-Cryst. Solids 156, 965 (1993).

[32] C. Oligschleger, C. Gaukel, H.R. Schober, J. Non-Cryst. Solids 250-252, 660 (1999).

[33] U. Buchenau, N. Nücker, A.J. Dianoux, Phys. Rev. Lett. 53, 2316 (1984).

[34] H.R. Schober, C. Gaukel, C. Oligschleger, Def. Diff. Forum 143-147, 723 (1997).

[35] H. Teichler, J. Non-Cryst. Solids, in press.

[36] W. Kob, J. Phys., Condens. Matter 11, R85 (1999).

[37] C. Donati, J.F. Douglas, W. Kob, S.J. Plimpton, P.H. Poole, S.C. Glotzer, Phys. Rev. Lett. 80, 2338 (1998).

[38] H. Ehmler, A. Heesemann, K. Rätzke, S. Rehders, F. Faupel, U. Geyer, Phys. Rev. Lett. 80, 4919 (1998).

[39] H.R. Schober, Solid State Commun. 119, 73 (2001). 